\title{
InSAC: A novel sub-nuclear body essential for Interleukin-6 and -10 RNA processing and stability
}

\author{
Sungwook Lee E Boyoun Park * \\ Department of Systems Biology, College of Life Science and Biotechnology, Yonsei University, Seoul 120-749, Korea
}

\begin{abstract}
Dysregulation of cytokine expression causes inflammatory diseases or chronic infection conditions. We have identified that Tat-activating regulatory DNA-binding protein-43 (TDP-43) is involved in cytokine RNA processing in order to promote an optimal immune response. The interaction of TDP-43 with spliceosomal components from the Cajal body leads to the formation of a novel sub-nuclear body called the Interleukin (IL)-6 and IL-10 Splicing Activating Compartment (InSAC). TDP-43 binds to the IL-6 and IL-10 RNAs in a sequence-dependent manner. In cell-based studies, we observed that lipopolysaccharide (LPS) stimulation induces the formation of the InSAC through TDP-43 ubiquitination, thereby influencing the processing and expression levels of IL-6 RNA. Moreover, TDP-43 knockdown in vivo results in a decrease in IL-6 production and its RNA splicing and stability. Thus, these findings demonstrate that the InSAC is linked to the activation and modulation of the immune response. [BMB Reports 2015; 48(5): 239-240]
\end{abstract}

The nucleus is compartmentalized into dynamic structural and functional sub-nuclear bodies; the Cajal body (CB) is an example of such a characterized sub-nuclear body. The CB is involved in the maturation of spliceosomal small nuclear ribonucleoproteins (snRNPs) and the biogenesis of the telomerase ribonuclear protein complex, which contributes to telomerase synthesis. Coilin is essential for $\mathrm{CB}$ integrity through its interaction with several $\mathrm{CB}$ components, such as snRNPs, survival of motor neuron (SMN), and Sm proteins. However, the precise molecular mechanism underlying the function of the $\mathrm{CB}$

*Corresponding author. E-mail: bypark@yonsei.ac.kr

http://dx.doi.org/10.5483/BMBRep.2015.48.5.060

Received 31 March 2015

Keywords: TDP-43, Cytokine, IL-6, Nuclear body, RNA processing

Abbreviations: NF- $\kappa$ B, nuclear factor kappa B; MEFs: mouse embryonic fibroblast cells; Cy3, Cyanine dye 3; RNA-FISH, RNA-fluorescence in situ hybridization

Perspective to: Sungwook Lee et al (2015), Identification of a subnuclear body involved in sequence-specific cytokine RNA processing, Nature Communications, 6:5791, doi: 10.1038/ncomms6791. and other sub-nuclear bodies remains to be elucidated.

TDP-43 is a highly conserved heterogeneous nuclear ribonucleoprotein (hnRNP), that has potential functions in RNA processing such as RNA transcription, splicing, trafficking, and degradation. We demonstrate that endogenous TDP-43 is enriched within spherical sub-nuclear bodies in macrophage and primary cell lines following LPS stimulation. Based on the results from various cell lines, we have also shown that the formation of the TDP-43 sub-nuclear body is related to the production of specific cytokines. Moreover, the sizes of TDP-43sub-nuclear foci are increased in a stimulation time-dependent manner, and those structures are similar to CBs. However, the localization of TDP-43 foci does not overlap with that of CBs and other sub-nuclear bodies, such as the nucleolus and promyelocytic leukemia (PML) bodies. Therefore, the TDP-43-enriched sub-nuclear body is novel structural compartment in the nucleus during the immune response.

Cytokines are important for the regulation of the immune response, however the key regulatory molecular complex related to cytokine RNA processing is largely unknown. Since the formation of TDP-43 foci is linked to cytokine production, we examined the molecular function of TDP-43 in cytokine RNA processing. TDP-43 depletion reduces the expression levels of IL- 6 and the quantity of its mRNA in response to LPS. In addition, accumulation of unspliced IL-6 pre-mRNA and impairment of IL-6 RNA processing are observed in TDP-43 depleted cells, indicating that TDP-43 is important for IL-6 RNA processing and stability. RNA immunoprecipitation (RIP), as well as RNA secondary structure prediction programs, reveal that TDP-43 binds to mouse IL- 6 pre-mRNA intron 2 containing a predicted stem-loop structure consisting of GC-rich palindromic repeats and a short spacer. However, TDP-43 knockdown does not affect the processing of tumor necrosis factor (TNF)- $\alpha$ RNA, which does not contain structure-specific sequences. Furthermore, both an RNA splicing assay based on the IL-6 minigene, and an RNA-FISH assay using Cy3-labeled IL-6 or IL-10 RNA, supported the notion that the TDP-43 sub-nuclear body is the essential nuclear site for IL-6 or IL-10 mRNA production and stability. As a result of this observation, it was named InSAC (the IL-6 and IL-10 Splicing Activating Compartment).

The TDP-43-mediated recruitment of essential spliceosomal components from $\mathrm{CBs}$ provides the molecular mechanism un- 




Fig. 1. Model of TDP-43-mediated InSAC function in IL-6 and IL-10 pre-mRNA processing. derlying InSAC formation in response to LPS. LPS stimulation induces post-translational modifications of TDP-43, such as oligomerization, fragmentation, and ubiquitination. In particular, we focused on the ubiquitination of TDP-43 and investigated the effect of UBE2E3, the ubiquitin-conjugating enzyme for TDP-43, on InSAC formation and IL-6 RNA processing. UBE2E3 depletion reduces TDP-43 ubiquitination and IL-6 expression levels. The decreased InSAC number and impaired IL-6 RNA processing are also observed in UBE2E3-depleted cells. Therefore, TDP-43 ubiquitination is required for InSAC formation in order to regulate IL-6 RNA processing. Using in vivo delivery reagents for TDP-43 siRNA, we observed reduced IL-6 mRNA production and defective RNA processing in the spleens of TDP-43-depleted mice, without any alteration in the amount of TNF- $\alpha$ RNA. In addition, in vivo, TDP-43-depleted mice show a decrease in IL-6 expression levels in serum, strongly supporting the role of TDP-43 as a critical regulator of IL-6 RNA processing.

In conclusion, this study identifies and characterizes the InSAC as a novel sub-nuclear site for IL-6 and IL-10 RNA processing. Our study demonstrates that IL-6 and IL-10 RNA processing is tightly controlled by TDP-43-mediated InSAC formation. TDP-43 serves as a scaffold protein in the InSAC and competitively recruits critical spliceosomal components from CBs in order to facilitate specific cytokine RNA processing (Fig. 1). In particular, TDP-43 interacts with RNAs containing GC-rich palindromic repeats along with the conserved 'ACU' sequence motifs within a short spacer region. These findings provide substantial new insight into the molecular mechanism of cytokine RNA processing. Additionally, IL-6 levels are usually elevated in the serum of patients suffering from hepatocellular carcinoma ( $\mathrm{HCC}$ ) and obesity. Interestingly, it has been reported that IL- $6^{-1-}$ mice develop fewer tumors than wild type controls, and that TDP-43-depleted xenograft mice reduce HCC volume and weight (Naugler et al (2007) Science 317: 121-124, DOI.10.1126/science.1140485, Park et al (2013) Hepatology 58: 182-191, DOI: 10.1002/hep.26310), indicating that TDP-43 overexpression may lead to accelerated IL-6 RNA production by recruiting a large amount of spliceosomal components that could lead to liver inflammation. Additionally, inflammatory diseases could be characterized by excessive TDP-43-induced InSAC formation. Taken together, these data provide further insight into the molecular pathology of various cancers, autoimmune diseases, and obesity, thereby contributes to the development of improved therapies for treating these diseases.

\section{ACKNOWLEDGEMENTS}

This study was supported by grants from the National Research Foundation of Korea (NRF) (2011-0015372) and from the National R\&D Program, Ministry of Health \& Welfare, Republic of Korea (1220060, HI14C2542). 